\title{
An approach to adjustment of relativistic mean field model parameters
}

\author{
Tuncay Bayram ${ }^{1, \mathrm{a}}$ and Serkan Akkoyun ${ }^{2, \mathrm{~b}}$ \\ 1 Department of Nuclear Energy Engineering, Sinop University, Sinop, Turkey \\ 2 Department of Physics, Cumhuriyet University, Sivas, Turkey
}

\begin{abstract}
The Relativistic Mean Field (RMF) model with a small number of adjusted parameters is powerful tool for correct predictions of various ground-state nuclear properties of nuclei. Its success for describing nuclear properties of nuclei is directly related with adjustment of its parameters by using experimental data. In the present study, the Artificial Neural Network (ANN) method which mimics brain functionality has been employed for improvement of the RMF model parameters. In particular, the understanding capability of the ANN method for relations between the RMF model parameters and their predictions for binding energies (BEs) of ${ }^{58} \mathrm{Ni}$ and ${ }^{208} \mathrm{~Pb}$ have been found in agreement with the literature values.
\end{abstract}

\section{Introduction}

The relativistic mean field (RMF) theory [1] with a small number of adjusted parameters has been used successfully in the last thirty years for predictions of various ground-state nuclear properties of nuclei [2-5]. It is a phenomenological model and its starts with a lagrangian density includes terms related with its interaction mechanism. Nucleons interact with each other via exchange of various mesons in this model. For this model, equation of motion can be obtained by applying least action principle on Lagrangian density. Thus set of coupled equations can be obtained (Dirac and KleinGordon like equations) and they are solved iteratively for calculations of nuclear properties of nuclei in the RMF model. For this, parameters included by lagrangian density such as masses of considered mesons, nucleonmeson coupling constants and self-couplings of mesons are needed. These parameters are fitted from experimental data. Therefore optimazing of these parameters is very important for correct prediction of the RMF model for nuclear properties of nuclei. In literature, many parameter sets of the RMF model have been introduced for correct predictions of nuclear properties of nuclei cover nuclidic chart (Please see Ref. [6] and references therein).

The Artificial Neural Network (ANN) method [7] are used successfully in many fields of science as in nuclear physics $[9,10]$. The ANN is known as a very powerful tool that is used when standard techniques fail to estimate the correlation between the variables. As we know from our previous studies $[8,11]$, the ANN is useful for evaluation of nuclear data. Therefore, it has been employed to check its understanding capability of relations between the RMF model parameters and their predictions on the ground-state BEs of ${ }^{58} \mathrm{Ni}$ and ${ }^{208} \mathrm{~Pb}$ nuclei in the present study.

\footnotetext{
a e-mail: t . bayram@ymail.com

b e-mail: serkan.akkoyun@gmail.com
}

\section{Method}

There can be found some different types of RMF model in literature. In the present paper, we have considered only non-linear version of the RMF model. For this reason, the non-linear RMF model is shortly described in this section. In the RMF model, nucleons interact with each other via exchange of various mesons. This exchanges determine average potential where nucleons move independently as relativistic particles. It starts with phenomenological lagrangian density given as

$$
\begin{array}{r}
L=\bar{\psi}\left(i \gamma^{\mu} \partial_{\mu}-M\right) \psi+\frac{1}{2} \partial^{\mu} \sigma \partial_{\mu} \sigma-U(\sigma) \\
-g_{\sigma} \bar{\psi} \sigma \psi-\frac{1}{4} \Omega^{\mu \nu} \Omega_{\mu \nu}+\frac{1}{2} m_{\omega}^{2} \omega^{\mu} \omega_{\mu} \\
-g_{\omega} \bar{\psi} \gamma^{\mu} \psi \omega_{\mu}-\frac{1}{4} \vec{R}^{\mu \nu} \vec{R}_{\mu \nu} \\
+\frac{1}{2} m_{\rho}^{2} \vec{\rho}^{\mu} \vec{\rho}_{\mu}-g_{\rho} \bar{\psi} \gamma^{\mu} \vec{\tau} \psi \vec{\rho}_{\mu} \\
-\frac{1}{4} F^{\mu \nu} F_{\mu \nu}-e \bar{\psi} \gamma^{\mu} \frac{1-\tau_{3}}{2} A_{\mu} \psi .
\end{array}
$$

In this equation, the meson fields are indicated by $\sigma$, $\omega$ and $\rho$ and their masses are denoted by $m_{\sigma}, m_{\omega}$ and $m_{\rho}$, respectively. The corresponding coupling constants for the mesons are $g_{\sigma}, g_{\omega}$ and $g_{\rho}$. The Dirac spinor $\psi$ denotes the nucleon with mass $M$. The photon field is denoted by $A_{\mu}$. The potential term $U(\sigma)$ in the Lagrangian density is important to provide the correct prediction of deformation and incompressibility of nuclei [12] and it includes $g_{2}$ and $g_{3}$ adjustable free parameters.

By applying the Euler-Lagrange equation for the Lagrangian density given above, the set of coupled equations (Dirac equation for nucleons and Klein-Gordon like equations for mesons) can be obtained. These equations can be solved iteratively for finite nuclei [13]. For obtaining ground-state properties of nuclei, the parameters $m_{\sigma}, g_{\sigma}, g_{\omega}, g_{\rho}, g_{2}$ and $g_{3}$ are needed and 
Table 1. The RMSE values of the ANN test results and the values of the NL3* parameters.

\begin{tabular}{lccl}
\hline Parameters & ${ }^{58} \mathrm{Ni}$ & ${ }^{208} \mathrm{~Pb}$ & $\mathrm{NL3}{ }^{*}$ \\
\hline$M_{\sigma}[\mathrm{MeV}]$ & 8.85 & 8.43 & 502.5742 \\
$g_{\sigma}$ & 0.43 & 0.20 & 10.0944 \\
$g_{\omega}$ & 0.32 & 0.15 & 12.8065 \\
$g_{\rho}$ & 0.03 & 0.08 & 4.5748 \\
$g_{2}\left[\mathrm{fm}^{-1}\right]$ & 0.40 & 0.31 & -10.8093 \\
$g_{3}$ & 1.32 & 1.31 & -30.1486 \\
\hline
\end{tabular}

they are fitted from experimental data. Mostly groundstate BEs of some spherical nuclei are used for fitting procedure. Also, charge radii of some spherical nuclei are used additionally.

The ANN is a mathematical method which mimics brain functionality [7]. It composed of many processing units called neurons in different layers which are input, hidden and output layers. Due to these layers, this type of ANN is the layered ANN. Input layer neurons receive the data from the environment and transmit to the hidden and then output layer. This data taken from the output is the solution of the problem. Because the data flows forward one-way, the ANN is named as the feed-forward ANN. The neurons are the processing units and connected to each other in different layers via adjustable weights. These weights provide communications between the neurons. The main purpose of the method is to determine the values of the weights by using given data. In the hidden layer, the data is activated by using an activation function. In the present study, one hidden layer and a sigmoid-like activation function has been used. The method is divided into mainly two steps. All data belonging to the problem is separated into two sets for each step. In the first step called training, the data including both input and desired output is given to the ANN. By training of the ANN, the weights are modified by using the data. The method generates its own outputs as close as possible to the desired data. The comparisons between desired data and ANN output data are performed by mean square error (MSE) function.

In the present study, we have tried to obtain the best non-linear RMF model parameters $M_{\sigma}, g_{\sigma}, g_{\omega}, g_{\rho}, g_{2}$ and $g_{3}$ for selected nuclei by using the ANN method. For this, $\mathrm{RMF}+\mathrm{ANN}$ hybrid calculations have been carried out. BEs of ${ }^{58} \mathrm{Ni}$ and ${ }^{208} \mathrm{~Pb}$ nuclei have been calculated within the framework of the RMF model by changing parameters step by step in a meaningful range first. Thus we have obtained theoretical BEs for different parameters values. In the second step, the obtained data has been used as inputs for the ANN method. Later, using experimental BEs of selected nuclei, the best non-linear RMF model parameters have been obtained in the test stage of the ANN method. Finally, the parameters obtained by using the ANN method have been used in the RMF calculations for the predictions of BEs of ${ }^{58} \mathrm{Ni}$ and ${ }^{208} \mathrm{~Pb}$ nuclei.

\section{Results and discussion}

After the training of data by using the ANN method properly, the test stage had been done and root mean square error (RMSE) values of $M_{\sigma}, g_{\sigma}, g_{\omega}, g_{\rho}, g_{2}$ and $g_{3}$ have been calculated. They have been listed in Table 1. Also, the parameters of the known NL3* effective force are shown in the last column of the table for comparison. As can be

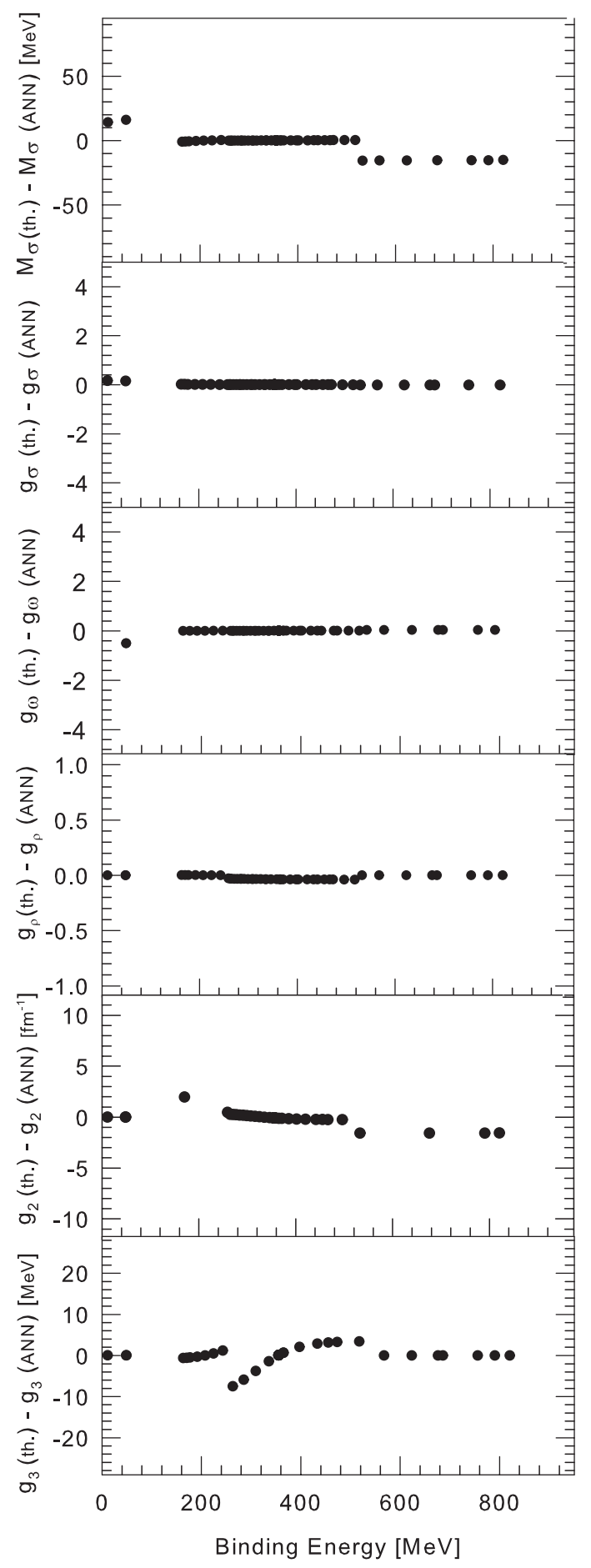

Figure 1. The differences between input and output data obtained from test stage for non-linear RMF model parameters $\left(M_{\sigma}, g_{\sigma}\right.$, $g_{\omega}, g_{\rho}, g_{2}$ and $g_{3}$ ) by considering ${ }^{58} \mathrm{Ni}$.

understood from the Table 1, RMSE values of the test stage of the ANN method for ${ }^{58} \mathrm{Ni}$ and ${ }^{208} \mathrm{~Pb}$ are at acceptable level. The RMSE values for $M_{\sigma}$ are seen as higher with respect to the others. However, it should be noted that $M_{\sigma}$ value is about $525 \mathrm{MeV}$.

The differences between input and output data obtained from test stage for non-linear the RMF model parameters by considering ${ }^{58} \mathrm{Ni}$ are shown in Fig. 1. Similar figures for ${ }^{208} \mathrm{~Pb}$ have been obtained but are not presented here. In this figure, small fluctuations are visible for $g_{2}$ and $g_{3}$ parameters with low BE values. $g_{2}$ and $g_{3}$ parameters are 
Table 2. The experimental and calculated ground-state BEs for ${ }^{58} \mathrm{Ni}$ and ${ }^{208} \mathrm{~Pb}$ nuclei. Units are in $\mathrm{MeV}$.

\begin{tabular}{lll}
\hline & ${ }^{58} \mathrm{Ni}$ & ${ }^{208} \mathrm{~Pb}$ \\
\hline Experiment [14] & 506.459 & 1636.430 \\
NL3* [5] & 654.622 & 1580.039 \\
This work & 464.209 & 1579.848 \\
\hline
\end{tabular}

non-linear self-coupling constants of $\sigma-$ mesons and they provide correct predictions of surface properties of nuclei. Therefore, one can expect these fluctuations for very low $\mathrm{BE}$ values.

After the test stage of ANN, the ground-state BEs of ${ }^{58} \mathrm{Ni}$ and ${ }^{208} \mathrm{~Pb}$ nuclei in the RMF model have been calculated by using optimal parameters. The results are listed in Table 2 together with the results of NL3* parameters set [5] and experimental data [14]. As can be understood from the Table 2, the results of the present study are close to the experimental data for ${ }^{58} \mathrm{Ni}$ and ${ }^{208} \mathrm{~Pb}$ nuclei. Furthermore the calculated $\mathrm{BE}$ in this work for ${ }^{58} \mathrm{Ni}$ is better than the those of the NL3* prediction. Thus, understanding capability of the ANN for non-linear relations between the RMF model parameters values and their predictions on $\mathrm{BEs}$ of ${ }^{58} \mathrm{Ni}$ and ${ }^{208} \mathrm{~Pb}$ nuclei is clearly seen.

\section{Conclusions}

The ANN method has been applied to check its understanding capability for non-linear relations between the RMF model parameters and their predictions on BEs of ${ }^{58} \mathrm{Ni}$ and ${ }^{208} \mathrm{~Pb}$ nuclei. The results show that the ANN method can be a powerful tool for understanding these non-linear relations. It should be noted that only BE has been considered in the present study. By increasing the number of nuclei taken into account as well as considering charge radii and single-particle levels this study could be expanded for more precise determination of ANN application success on the RMF model parameters.

This work has been supported by the Scientific Research Council of Turkey (TÜBITTAK) under Project No. 115F291.

\section{References}

[1] J.D. Walecka, Ann. Phys. 83, 491 (1974)

[2] P. Ring, Prog. Theor. Phys. 37, 193 (1996)

[3] D. Vretenar, A.V. Afanasjev, G.A. Lalazissis, P. Ring, Phys. Rep. 409, 101 (2005).

[4] J. Meng, H. Toki, S.-G. Zhou, S.-Q. Zhang, W.-H. Long, L.-S. Geng, Prog. Part. Nucl. Phys. 57, 470 (2006)

[5] T. Bayram, A.H. Yilmaz, Mod. Phys. Lett. A 28, 1350068 (2013)

[6] G.A. Lalazissis, S. Karatzikos, R. Fossion, D. Pena Artega, A.V. Afanasjev, P. Ring, Phys. Lett. B 671, 36 (2009)

[7] S. Haykin, Neural Networks: a Comprehensive Foundation (Prentice-Hall, Delhi, 1999)

[8] T. Bayram, S. Akkoyun, S.O. Kara, Ann. Nucl. Energy 63, 172 (2014)

[9] F. Haddad, K. Hagel, J. Li, N. Mdeiwayeh, J.B. Natowitz, R. Wada, B. Xiao, C. David, M. Freslier, J. Aichelin, Phys. Rev. C 55, 1371 (1997)

[10] M.E. Medhat, Ann. Nucl. Energy 45, 73 (2012)

[11] S. Akkoyun, T. Bayram, Int. J. Mod. Phys. E 23, 1450064 (2014)

[12] J. Boguta, A.R. Bodmer, Nucl. Phys. A 292, 413 (1977)

[13] P. Ring, Y.K. Gambhir, G.A. Lalazissis, Comp. Phys. Commun. 105, 77 (1997)

[14] G. Audi, A.H. Wapstra, C. Thibault, Nucl. Phys. A 729, 337 (2003) 